\title{
A New Single-row Cruciate Suture Anchor Repair for Rotator Cuff Tears
}

\author{
Jarrad Stevens $^{1}$, Louise Richardson ${ }^{2}$, Tony Sobol ${ }^{1}$, Martin Richardson ${ }^{1,2}$
}

${ }^{1}$ Department of Surgery, University of Melbourne, Australia

${ }^{2}$ Epworth Health Care, Musculoskeletal Clinical Institute, Victoria, Australia

\section{Abstract}

\section{Publication History:}

Received: November 03, 2017

This paper will present the technique for a novel suture anchor configuration used in the management Accepted: February 12, 2018 of rotator cuff tears. The senior author (MR) developed this technique, involving single-row cruciate Published: February 14, 2018 sutures, to increase the footprint contact surface area while creating a desirable tensile pressure between the suture and tendon. Biomechanical studies of this technique have demonstrated a mean footprint contact area of $253 \mathrm{~mm} 2$ while maintaining a contact pressure of $0.78 \mathrm{Mo}$. In addition to its biomechanical validity, this simple configuration is easy to master, amenable to healing and not prone to cut out. The use of a single-row anchor also decreases the comparable cost with double-row techniques.

\section{Keywords:}

Shoulder, Tendon, Footprint, Contact area, Repair, Technique, Surgery, Cruciate

\section{Introduction}

Options for surgical management of rotator cuff disease include: subacromial decompression and bursectomy, debridement of partial tears and surgical repair of partial or full thickness tears. The standard technique for a repairable rotator cuff tear involves suture anchor placement into an osseous bed prepared in the humeral tuberosity, with a variety of different options for suture configuration. Multiple anchors may be required for acceptable repair

While many factors influence the outcome of rotator cuff surgery, the most common complication is structural failure of the tendon repair [1]. Better patient-reported outcomes have been shown in patients with structurally intact repairs [2,3], with an improved range of motion and strength compared to patients with structural retears [4-6]. The weakest region of the tendon repair is the suture-tendon interface, which typically becomes a problem in the case of pullout of the suture through the tendon [7]. This complication prompted the senior author to explore single-row suture techniques, which provide a large footprint contact area for the bone tendon interface, as well as creating adequate contact pressure between the suture and tendon. Clinical experience has shown that this technique is amenable to healing and not prone to cutting out. In vitro studies also support the biomechanical efficacy of this technique $[8,11]$.

The section below presents an intra-operative description of this technique.

\section{Indications}

Arthroscopic or mini-open evaluation of rotator cuff tears deemed to be appropriate for repair with the use of suture anchors.

\section{Technique}

Mini-open surgery is performed under general anaesthesia with optional regional nerve blocks. The patient is placed in the lateral decubitus position with the operative arm placed in traction - typically $10-15 \mathrm{lbs}(4.5-7 \mathrm{~kg})$ (Figure 1). Appropriate antiseptic preparation and draping is performed. The patient receives prophylactic intravenous antibiotic before the skin incision. A straight longitudinal incision measuring approximately $3-4 \mathrm{~cm}$ in length is placed at the midportion of the lateral acromion. A deltoid split approach is utilised to present the rotator cuff tear for assessment and treatment, typically after arthroscopic acromioplasty and bursectomy.

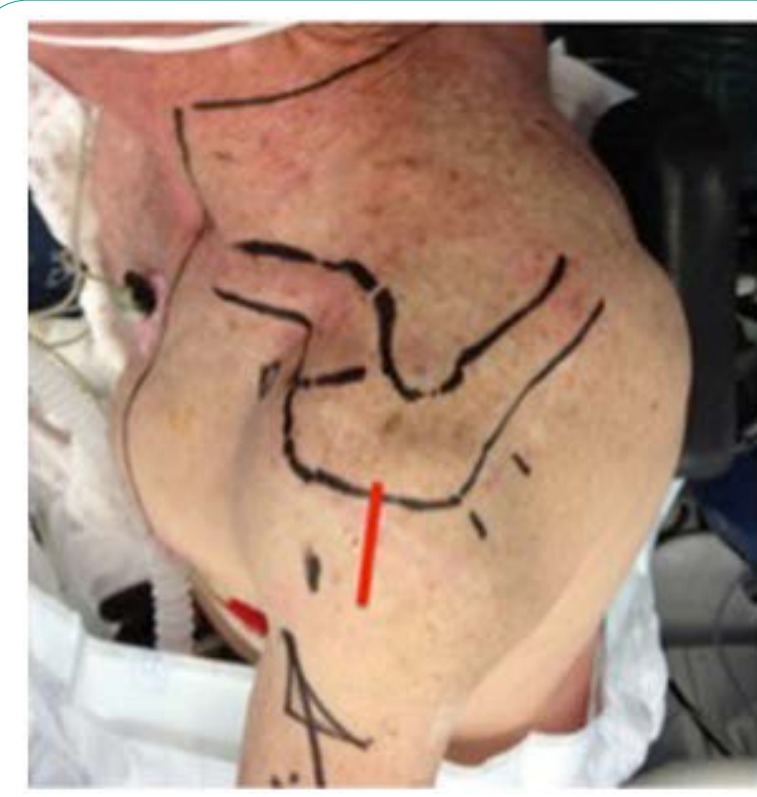

Figure 1: Landmarks and the site for incision.

Sutures are placed into the opposing torn ends of the rotator cuff defect using No.1 Vicryl; this will enable manual retraction and increase manoeuvrability of the torn tendons. A Cobb elevator is used to free adhesions on the bursal and articular sides of the rotator cuff, minimizing tension in the repaired tendon. The surface of the tuberosity is decorticated with the aid of a low speed (8000rpm) burr exposing a cancellous footprint. A suture anchor with two loaded sutures (four strands) is selected. The author prefers non-absorbable suture anchors with No.2 Fiber Wire or Ethibond braided sutures. The anchor awl is introduced into the central midpoint of the footprint at Deadman's angle, as described by Burkhart [9].

"Corresponding Author: Dr. Jarrad Stevens, Department of Surgery, University of Melbourne, Australia; Email: drjarradstevens@hotmail.com

Citation: Stevens J, Richardson L, Sobol T, Richardson M (2018) Surgical Technique: A New Single-row Cruciate Suture Anchor Repair for Rotator Cuff Tears. Int J Surg Surgical Porced 3: 129. https://doi.org/10.15344/2456$4443 / 2018 / 129$

Copyright: (c) 2017 Stevens et al. This is an open-access article distributed under the terms of the Creative Commons Attribution License, which permits unrestricted use, distribution, and reproduction in any medium, provided the original author and source are credited. 
Citation: Stevens J, Richardson L, Sobol T, Richardson M (2018) Surgical Technique: A New Single-row Cruciate Suture Anchor Repair for Rotator Cuff Tears. Int J Surg Surgical Porced 3: 129. https://doi.org/10.15344/2456-4443/2018/129

Page 2 of 3

The cruciate suture repair consists of two overlapping stitches, oriented at $45^{\circ}$ to the tendon, acting to increase tendon-bone contact and prevent tendon slippage. With the use of a mayo needle, the single-row cruciate stitch is placed using an opposing near-far stitch. The first stitch is placed far ( $12 \mathrm{~mm}$ from the torn tendon edge). The second limb of this stitch is placed into the opposite tendon edge in the near position ( $4-5 \mathrm{~mm}$ from the torn edge seen in Figure 2). The technique is then repeated with the second suture from the anchor, the tendon can now be laid flat on the bone with the assistance of the tacking sutures (Figure 3). The sutures from the anchor are now tied creating a cruciate stich (Figures 4 and 5). The initial knot tied for each stitch is a sliding half-hitch, this is locked by three half-hitch knots tied at alternating posts. Knots are positioned immediately above the repaired tendon. If the repair requires, two side by side single row cruciate sutures can be placed (Figure 6).

Standard closure techniques are performed and post operatively the patient is placed into a broad arm sling. Typically, this operation can be performed as a day or overnight stay procedure. Pendulum activities are encouraged for the first 4-6 weeks, followed by a graduated return to function and physiotherapy-led rehabilitation program.

\section{Discussion}

This paper outlines the single-row cruciate suture technique. Traditional single-row suture configurations can pass through poor

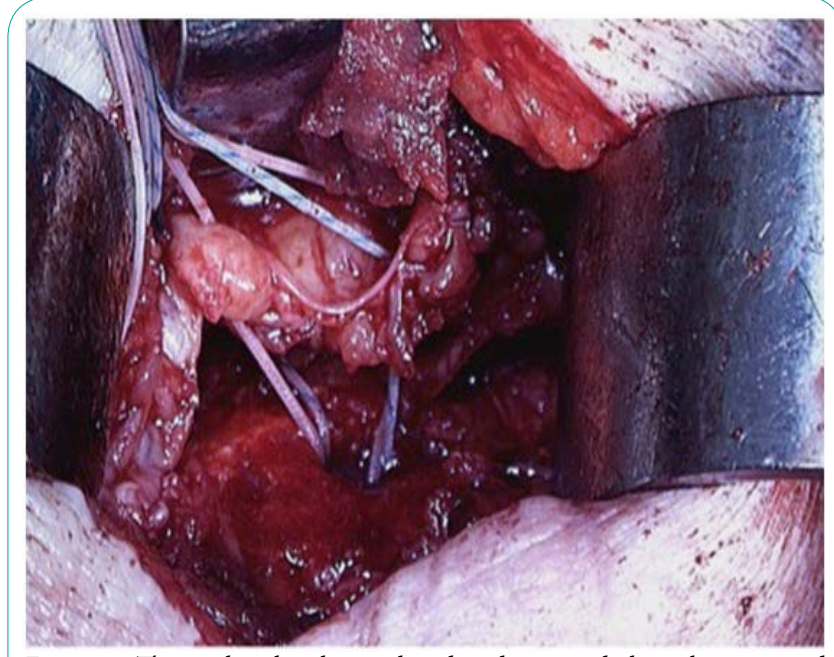

Figure 2: The anchor has been placed and 4 strands have been passed through the tendon as described.

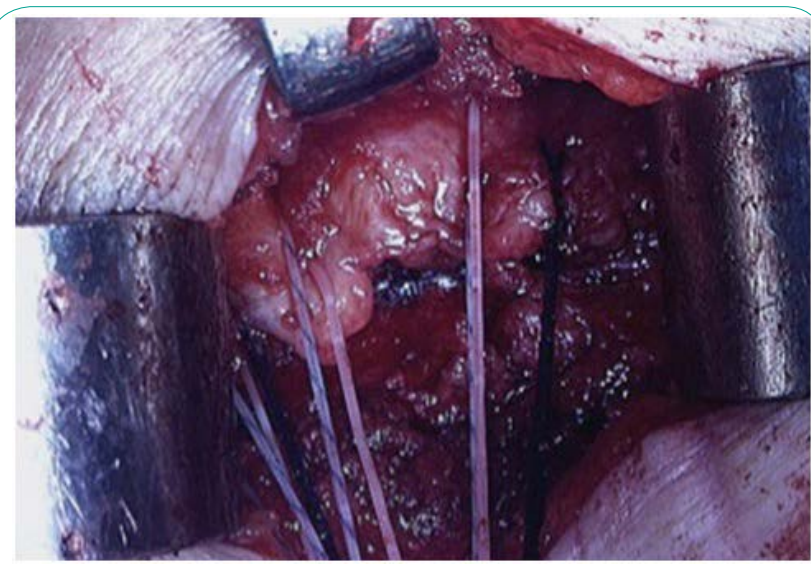

Figure 3: The tendon can now be manoeuvred into place and assessed. quality tendon edges, thereby potentially adding to adverse tendon suture pull-out. The cruciate technique, however, passes through both good quality tendon (far stitch) for increased pullout strength, and marginal tendon (near stitch) for apposition. While double-row techniques may provide superior contact area and ultimate tensile strength compared to traditional single-row repairs [10], they are associated with higher material expenses and prolonged operating time and are technically more challenging for the surgeon.

The single-row cruciate suture technique has been validated in vitro and published by the lead surgeon (MR) [8]. In an animal study utilising infraspinatus tendons from lamb's shoulders, the cruciate

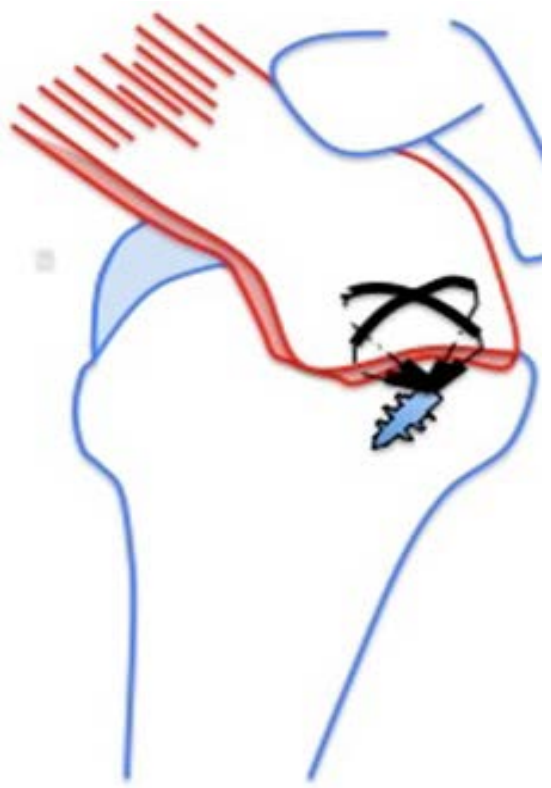

Figure 4: Near far cross cruciate single-row anchor repair.

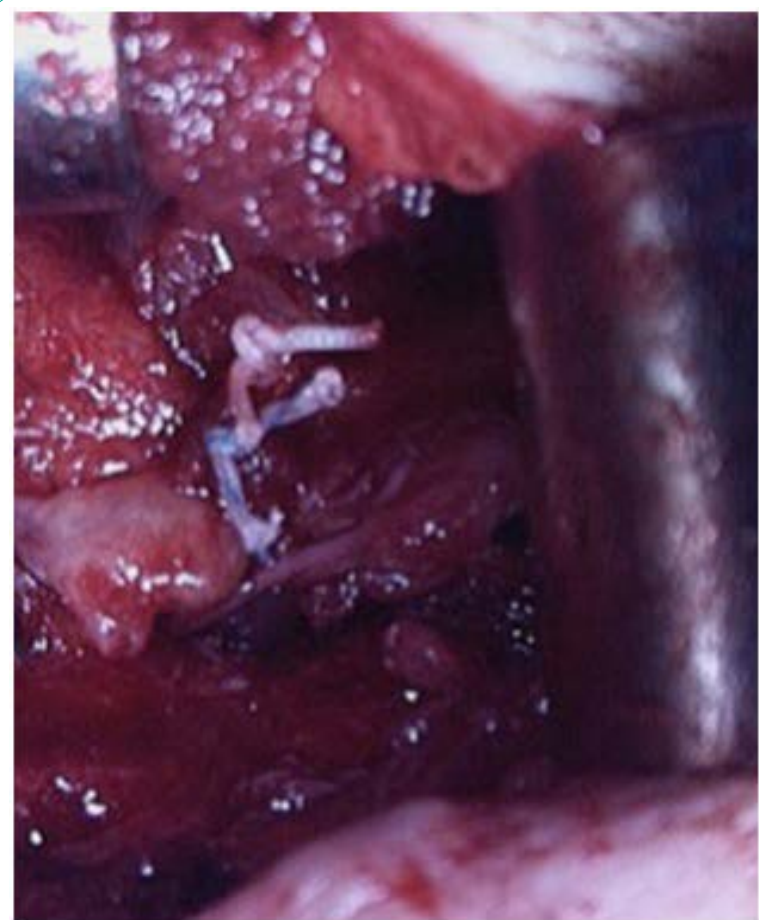

Figure 5: The cruciate suture is tied and the repair inspected. 


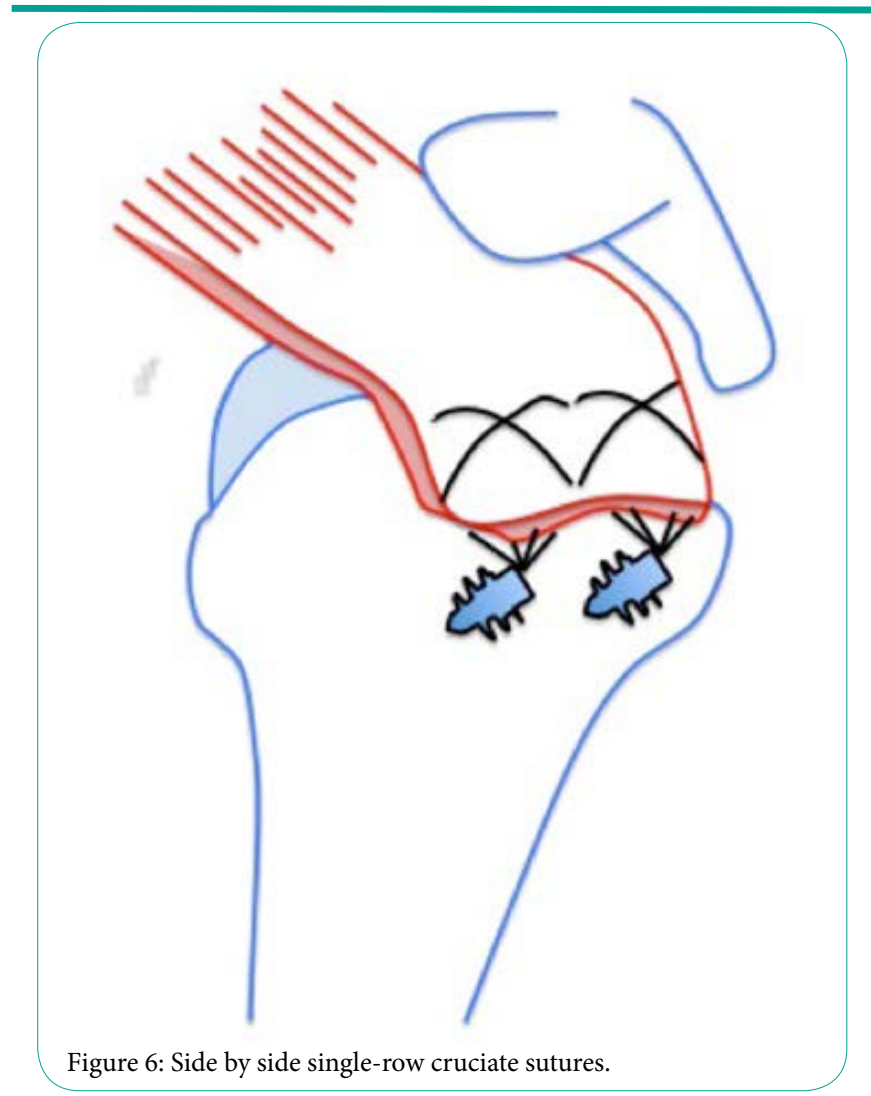

suture repair established a significantly greater footprint contact area compared to the Mason-Allen repair (mean difference $101 \mathrm{~mm}^{2}, \mathrm{p}=$ 0.003). The average footprint contact pressure of the cruciate suture repair $(0.78 \mathrm{MPa})$ was similar to that of the Mason-Allen $(0.74 \mathrm{MPa})$ and double-row repairs $(0.79 \mathrm{MPa})$. The ultimate tensile strength of the cruciate suture repair was significantly greater than that of the transosseous repair (mean difference 62.4 N, p = 0.002).

The authors therefore concluded that cruciate suture repair may improve strength and healing at the repaired tendon rotator cuff insertion compared to other single-row repair techniques. Further animal studies have demonstrated Gap formation in the single-row cruciate suture repair is significantly lower than that of the MasonAllen repair (mean difference $=0.6 \mathrm{~mm}, \mathrm{P}=0.009$ ) and no different from that of the Suture Bridge repair $(P>0.05)$ at 200 cycles [11].

\section{Conclusion}

Compared to double-row techniques, single-row procedures can utilise half the number of suture anchors, adding to its cost efficacy and promoting sustainable resource management. Furthermore, the marginal difference in mechanical performance of the double-row repair has not been definitively shown to directly translate to superior clinical performance $[12,13]$. In contrast, the single-row cruciate repair technique described in this paper is a simple configuration, which is easy to master with biomechanical properties validated by in vitro testing $[8,11]$.

\section{Competing Interests}

The authors declare that they have no competing interests.

\section{References}

1. Gazielly DF, Gleyze P, Montagnon C (1994) Functional and anatomical results after rotator cuff repair. Clin Orthop Relat Res 304: 43-53.

2. DeFranco MJ, Bershadsky B, Ciccone J, Yum JK, lannotti JP (2007) Functional outcome of arthroscopic rotator cuff repairs: a correlation of anatomic and clinical results. J Shoulder Elbow Surg 16: 759-765.

3. Klepps S (2004) Prospective evaluation of the effect of rotator cuff integrity on the outcome of open rotator cuff repairs. Am J Sports Med 32: 17161722.

4. Harryman DT, Mack LA, Wang KY, Jackins SE, Richardson ML, et al. (1991) Repairs of the rotator cuff. Correlation of functional results with integrity of the cuff. J Bone Joint Surg Am 73: 982-989.

5. Huijsmans PE, Pritchard MP, Berghs BM, van Rooyen KS, Wallace AL, et al. (2007) Arthroscopic rotator cuff repair with double-row fixation. J Bone Joint Surg Am 89: 1248-1557.

6. Lafosse L, Brozska R, Toussaint B, Gobezie R (2007) The outcome and structural integrity of arthroscopic rotator cuff repair with use of the doublerow suture anchor technique. J Bone Joint Surg Am 89: 1533-1541.

7. Baleani M, Ohman C, Guandalini L, Rotini R, Giavaresi G, et al. (2006) Comparative study of different tendon grasping techniques for arthroscopic repair of the rotator cuff. Clin Biomech 21: 799-803.

8. Caldow J, Richardson M, Balakrishnan S, Sobol T, Lee P, et al. (2015) A cruciate suture technique for rotator cuff repair. Knee Surg Sports Traumatol Arthrosc 23: 619-626.

9. Burkhart SS (2009) Suture anchor insertion angle and the dead man theory. Arthroscopy 25: 1365

10. Ma CB, Comerford L, Wilson J, Puttlitz CM (2006) Biomechanical evaluation of arthroscopic rotator cuff repairs: double-row compared with single-row fixation. J Bone Jt Surg Am 88: 403-410.

11. Huntington L, Richardson M, Sobol T, Caldow J, Ackland D (2017) Load response and gap formation in a single-row cruciate suture rotator cuff repair. ANZ J Surg 87: 483-487.

12. Burks RT, Crim J, Brown N, Fink B, Greis PE (2009) A prospective randomized clinical trial comparing arthroscopic single- and doublerow rotator cuff repair: magnetic resonance imaging and early clinical evaluation. Am J Sports Med 37: 674-682.

13. Reardon DJ, Maffulli N (2007) Clinical evidence shows no difference between single- and double-row repair for rotator cuff tears. Arthroscopy 23: $670-673$. 